\title{
Non-Linear Water Wave Equation Time Series Formulated Using Velocity Equation as the Result of Laplace Equation
}

\author{
Syawaluddin Hutahaean, and Hendra Achiari
}

syawaluddin@ocean.itb.ac.id, and h_achiari@ocean.itb.ac.id, Ocean Engineering Program, Faculty of Civil and Environmental Engineering - Bandung Institute of Technology (ITB) - INDONESIA

\section{ABSTRACT}

This article developed a model of time series wave where during the formulation velocity equations as the result of Laplace theory was done. As the result of this method, the continuity equation was substituted to momentum equation, or in other words momentum equation produces a velocity controlled by continuity equation, since Laplace equation comes from continuity equation. Using this method, the concept of calculation velocity at the momentum equation was also developed where calculation velocity is equal to average velocity of the depth of the Airy long wave equation.

Key word: Continuity equation, Laplace equation, Momentum equation, Nonlinear wave.

\section{INTRODUCTION}

Velocity at a momentum equation is a velocity at any position on a vertical axis. In a numerical calculation using discrete method, finite element and finite difference method, a calculation can be done for some velocities along the vertical axis. However, in very shallow water, it is easier to do the calculation using a representative velocity, or a calculation velocity, so that in a momentum equation, there is only velocity on one depth position. This calculation velocity is known as depth-averaged velocity on Airy long wave equation introduced by Fenton [1].

The velocity produced by momentum equation should meet continuity equation. Therefore, there should be the role of continuity equation at the momentum equation. In this research, the role of continuity equation is substituted to momentum equation through driving force by working on the vertical direction of velocity equation as a result of Laplace equation solution

\section{VELOCITY RELATION AS A RESULT OF LAPLACE EQUATION SOLUTION}

The velocity of particle the horizontal and vertical direction based on Hutahaean [2],[3],[4] is defined as,

$u=G e^{k h} \beta(z)\left(k \sin k x-\frac{1}{2 h} \frac{\partial h}{\partial x} \cos k x\right) \sin \sigma t$

The velocity of particle in vertical -direction

$w=-G e^{k h} k \beta_{1}(z) \cos k x \sin \sigma t$ where

$$
\begin{aligned}
& G=\text { wave constant } \\
& k=\text { wave number } \\
& \sigma=\text { angle velocity } \\
& h=\text { water depth } \\
& \frac{\partial h}{\partial x}=\text { slope of water bottom } \\
& \beta(z)=\alpha e^{k(h+z)}+e^{-k(h+z)}, \\
& \beta_{1}(z)=\alpha e^{k(h+z)}-e^{-k(h+z)} \\
& \left.\alpha=\frac{1}{2} \mid \frac{\partial h}{1+\frac{\partial x}{\partial h}}+\frac{\partial-\frac{\partial h}{\partial x}}{1+\frac{\partial h}{\partial x}}\right)
\end{aligned}
$$

With the characteristics, $\frac{\partial k(h+z)}{\partial x}=0$ and $\frac{\partial k h}{\partial x}=0$, then

$$
\frac{\partial \beta(z)}{\partial x}=\frac{\partial \beta_{1}(z)}{\partial x}=\frac{\partial e^{k h}}{\partial x}=0 \text { and } \frac{\partial k}{\partial x}=-\frac{k}{h} \frac{\partial h}{\partial x} .
$$

Another characteristic is $\frac{\partial G}{\partial x}=\frac{G}{2 h} \frac{\partial h}{\partial x}$.

From equation (2.1) a relation can be made,

$u=\frac{\beta(z)}{\beta(\eta)} u_{\eta}$

And also, 


$$
\frac{\partial u}{\partial t}=\frac{\beta(z)}{\beta(\eta)} \frac{\partial u_{\eta}}{\partial t}-\frac{\beta(z)}{\beta^{2}(\eta)} \beta_{1}(\eta) k u_{\eta} \frac{\partial \eta}{\partial t}
$$

\section{III.THE CONCEPT OF CALCULATION VELOCITY}

At the continuity equation and momentum equation, velocity is a function of $z$, where $z$ is the vertical axis as shown on Fig 3.1, so, in a water column from the bottom to the surface of the water, there will be plenty of velocity. Calculation will be much easier if it is done using a representative velocity on a water column. This velocity is called depth calculation velocity. It was developed for the first time during the formulation of Airy long wave equation developed by Dean [5], called depth-averaged velocity, i.e.

$U=\frac{1}{\beta_{u} H} \int_{-h}^{\eta} u d z$

In the equation (3.1), the value of depth-averaged velocity is determined by the value of integration coefficient $\beta_{u}$. The bigger value of $\beta_{u}$, makes the smaller value of $U$, where this shows that a value of integration coefficient corresponds to a velocity at a depth of $z$.

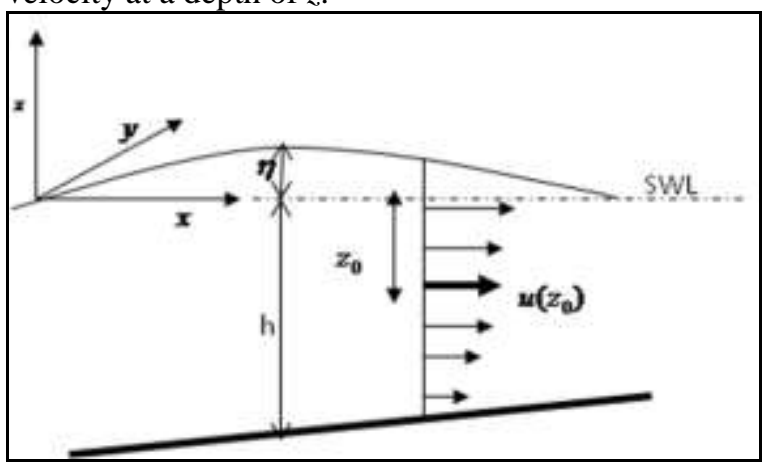

Figure 3.1. Depth-averaged Velocity and axis system

Equation (3.1) can be written as equation for integration coefficient, $\beta_{u}$ defined as

$\beta_{u}=\frac{1}{U H} \int_{-h}^{\eta} u d z$. If the depth-averaged velocity of

$U$ is a velocity at $z=z_{0}$, or $U=u\left(x, z_{0}, t\right)$, then $\beta_{u}=\frac{1}{u\left(z_{0}\right) H} \int_{-h}^{\eta} u d z$.

Using the form of velocity equation in the direction of horizontal $x$ as equation (2.1), then

$\beta_{u}=\frac{1}{\beta\left(z_{0}\right) H} \int_{-h}^{\eta} \beta(z) d z=\frac{\beta_{1}(\eta)-(\alpha-1)}{k \beta\left(z_{0}\right) H}$
Table 3.1 shows the result of $\beta_{u}$ calculation for various wave periods, for $z_{0}=-0.40 h$. The result of the calculation shows that at $z_{0}=-0.40 h$, the value of $\beta_{u}$ was obtained which is very close to 1 . This shows that depth-averaged velocity is a velocity at a depth of $z=z_{0}$.

Table 3.1. The value of $\beta_{u}$, at $z_{0}=-0.40 \mathrm{~h}$,

\begin{tabular}{|c|c|c|c|c|c|c|}
\hline $\begin{array}{c}\mathbf{H} \\
(\mathrm{m})\end{array}$ & $\begin{array}{l}T=6 \\
(\mathrm{sec})\end{array}$ & $\begin{array}{l}\boldsymbol{T}=\mathbf{8} \\
(\mathrm{sec}) \\
\end{array}$ & $\begin{array}{c}T=10 \\
(\mathrm{sec})\end{array}$ & $\begin{array}{c}T=12 \\
(\mathrm{sec})\end{array}$ & $\begin{array}{c}T=14 \\
(\mathrm{sec})\end{array}$ & $\begin{array}{c}T=16 \\
(\mathrm{sec})\end{array}$ \\
\hline 15 & 1.021 & 0.999 & 0.997 & 0.998 & 0.998 & 0.999 \\
\hline 14 & 1.017 & 0.999 & 0.998 & 0.998 & 0.998 & 0.999 \\
\hline 13 & 1.014 & 0.999 & 0.998 & 0.998 & 0.999 & 0.999 \\
\hline 12 & 1.011 & 0.999 & 0.999 & 0.999 & 0.999 & 0.999 \\
\hline 11 & 1.009 & 1 & 0.999 & 0.999 & 0.999 & 0.999 \\
\hline 10 & 1.008 & 1 & 0.999 & 0.999 & 1 & 1 \\
\hline 9 & 1.007 & 1.001 & 1 & 1 & 1 & 1 \\
\hline 8 & 1.007 & 1.001 & 1 & 1 & 1 & 1 \\
\hline 7 & 1.007 & 1.002 & 1.001 & 1 & 1 & 1 \\
\hline 6 & 1.007 & 1.003 & 1.001 & 1.001 & 1.001 & 1 \\
\hline 5 & 1.008 & 1.003 & 1.002 & 1.001 & 1.001 & 1.001 \\
\hline 4 & 1.009 & 1.004 & 1.003 & 1.002 & 1.001 & 1.001 \\
\hline 3 & 1.011 & 1.005 & 1.003 & 1.002 & 1.002 & 1.001 \\
\hline 2 & 1.013 & 1.007 & 1.004 & 1.003 & 1.002 & 1.002 \\
\hline 1 & 1.017 & 1.009 & 1.006 & 1.004 & 1.003 & 1.002 \\
\hline
\end{tabular}

In this paper, the concept of depth-averaged velocity is used with reversed procedure, i.e. the position of $z_{0}$ is set, and then integration coefficient is calculated using equation (3.2). The use of $z_{0}=-0.40 h$ will give $\beta_{u}$ integration coefficient, very close to 1 .

With the inversion of the procedure, the velocity integration is defined as $\int_{-h}^{\eta} u d z=\beta_{u} U H$ where $U=u_{z_{0}}$. Integration coefficient $\beta_{u}$, is calculated by using equation (3.2) and a new integration coefficient is defined as,

$\beta_{u H}=\beta_{u} H=\frac{\beta_{1}(\eta)-(\alpha-1)}{k \beta\left(z_{0}\right)}$

Therefore, $\int_{-h}^{\eta} u d z=\beta_{u H} U$

With equation (3.4), the integration of continuity equation to vertical axis can be done easily. 


\section{IV.THE FORMULATION OF MOMENTUM EQUATION}

As the basic momentum equation, Euler momentum equation developed by Dean [5] is used. The momentum equation is for the flow at $(x, z)$ plane where $x$ axis is horizontal axis and $z$ axis is vertical axis, consisting of two equations, i.e. momentum equation in horizontal $x$ direction and momentum equation in vertical $z$ direction, respectively

$$
\begin{aligned}
& \frac{\partial u}{\partial t}+u \frac{\partial u}{\partial x}+w \frac{\partial u}{\partial z}=-\frac{1}{\rho} \frac{\partial p}{\partial x} \\
& \frac{\partial w}{\partial t}+u \frac{\partial w}{\partial x}+w \frac{\partial w}{\partial z}=-\frac{1}{\rho} \frac{\partial p}{\partial z}-g
\end{aligned}
$$

Where $u=u(x, z, t)$ is the velocity in horizontal- $x$ direction, $w=w(x, z, t)$ velocity in vertical- $z$ direction, $p$ is pressure on water particle, $g$ is gravitational velocity, $\rho$ is water density and $t$ is time.

At the convective equation, the characteristic of irrotational flow is done where $\frac{\partial w}{\partial x}=\frac{\partial u}{\partial z}$. Hence based Hutahean [6], momentum $x$ dan $z$ equations become

$$
\begin{aligned}
& \frac{\partial u}{\partial t}+\frac{1}{2} \frac{\partial}{\partial x}(u u+w w)=-\frac{1}{\rho} \frac{\partial p}{\partial x} \\
& \frac{\partial w}{\partial t}+\frac{1}{2} \frac{\partial}{\partial z}(u u+w w)=-\frac{1}{\rho} \frac{\partial p}{\partial z}-g
\end{aligned}
$$

\subsection{The Formulation of Driving Force}

Equation (4.4) is written as driving force equation in vertical $z-\frac{1}{\rho} \frac{\partial p}{\partial z}$ direction, i.e.

$$
-\frac{1}{\rho} \frac{\partial p}{\partial z}=\frac{\partial w}{\partial t}+\frac{1}{2} \frac{\partial}{\partial z}(u u+w w)+g
$$

Then, it is multiplied by $d z$ and integrated into vertical $z$ axis,

$$
-\frac{1}{\rho} \int_{z}^{\eta} d p=\int_{z}^{\eta} \frac{\partial w}{\partial t} d z+\frac{1}{2} \int_{z}^{\eta} \partial(u u+w w)+\int_{z}^{\eta} g d z
$$

where $\eta=\eta(x, t)$ is water surface elevation. Integration is completed and dynamic surface boundary condition, $p_{\eta}=0$, is done

$$
\frac{p}{\rho}=\int_{z}^{\eta} \frac{\partial w}{\partial t} d z+\frac{1}{2}\left(u_{\eta} u_{\eta}+w_{\eta} w_{\eta}\right)
$$

$$
-\frac{1}{2}(u u+w w)+g(\eta-z)
$$

Equation (4.5) is pressure equation experienced by water particle. The driving force is pressure difference, so to obtain driving force at horizontal- $x$ direction; equation (4.5) is differentiated in $x$ direction.

$$
\begin{aligned}
\frac{1}{\rho} \frac{\partial p}{\partial x}= & \frac{\partial}{\partial x} \int_{z}^{\eta} \frac{\partial w}{\partial t} d z+\frac{1}{2} \frac{\partial}{\partial x}\left(u_{\eta} u_{\eta}+w_{\eta} w_{\eta}\right) \\
& -\frac{1}{2} \frac{\partial}{\partial x}(u u+w w)+g \frac{\partial \eta}{\partial x}
\end{aligned}
$$

Substitute equation (4.6) to horizontal- $x$ momentum equation, equation (4.3),

$$
\begin{aligned}
& \frac{\partial u}{\partial t}+\frac{1}{2} \frac{\partial}{\partial x}(u u+w w)=-\frac{\partial}{\partial x} \int_{z}^{\eta} \frac{\partial w}{\partial t} d z \\
& -\frac{1}{2} \frac{\partial}{\partial x}\left(u_{\eta} u_{\eta}+w_{\eta} w_{\eta}\right)+\frac{1}{2} \frac{\partial}{\partial x}(u u+w w)-g \frac{\partial \eta}{\partial x}
\end{aligned}
$$

The second term on the left-hand side and the third term on the right hand side are eliminated each other

$$
\frac{\partial u}{\partial t}=-\frac{\partial}{\partial x} \int_{z}^{\eta} \frac{\partial w}{\partial t} d z-\frac{1}{2} \frac{\partial}{\partial x}\left(u_{\eta} u_{\eta}+w_{\eta} w_{\eta}\right)-g \frac{\partial \eta}{\partial x}
$$

In equation (4.7), there is still an integral that should be completed, i.e. integral in the first term of the right hand side. Equation $\frac{\partial}{\partial x} \int_{z}^{\eta} \frac{\partial w}{\partial t} d z$ is obtained by integrating continuity equation that will be done in the next section.

4.2 Equation for $\frac{\partial}{\partial x} \int_{z}^{\eta} \frac{\partial w}{\partial t} d z$

Huatahean [7] completed the integration of $\frac{\partial}{\partial x} \int_{z}^{\eta} \frac{\partial w}{\partial t} d z$ by integrating continuity equation. In this paper, the integration is completed using velocity equation from the solution of Laplace equation. From the velocity equation, the equation (2.2), become

$$
\begin{aligned}
& \frac{\partial w}{\partial t}=-G k e^{k h} \beta_{1}(z) \sigma \cos k x \cos \sigma t \text { so } \\
& \int_{z}^{\eta} \frac{\partial w}{\partial t} d z=-G e^{k h}(\beta(\eta)-\beta(z)) \sigma \cos k x \cos \sigma t
\end{aligned}
$$


$\frac{\partial}{\partial x} \int_{z}^{\eta} \frac{\partial w}{\partial t} d z=G e^{k h}(\beta(\eta)-\beta(z)) \sigma k \sin k x \cos \sigma t t$

$-\frac{\partial G}{\partial x} e^{k h} \sigma(\beta(\eta)-\beta(z)) \cos k x \cos \sigma$

Therefore

$$
\begin{aligned}
\frac{\partial}{\partial x} \int_{z}^{\eta} \frac{\partial w}{\partial t} d z & =G e^{k h}(\beta(\eta)-\beta(z)) \\
& \mathrm{X} \sigma\left(k \sin k x-\frac{1}{2 h} \frac{\partial h}{\partial x} \cos k x\right) \cos \sigma t
\end{aligned}
$$

From the velocity equation, equation (2.1) so

$\frac{\partial u}{\partial t}=G e^{k h} \beta(z) \sigma\left(k \sin k x-\frac{1}{2 h} \frac{\partial h}{\partial x} \cos k x\right) \cos \sigma t$

Substitute equation $\frac{\partial u}{\partial t}$ to equation $\frac{\partial}{\partial x} \int_{z}^{\eta} \frac{\partial w}{\partial t} d z$ the last,

$\frac{\partial}{\partial x} \int_{z}^{\eta} \frac{\partial w}{\partial t} d z=\left(\frac{\partial u}{\partial t}\right)_{z=\eta}-\frac{\partial u}{\partial t}$

Equation (2.4) was done at $z=\eta$,

$$
\begin{aligned}
& \left(\frac{\partial u}{\partial t}\right)_{z=\eta}=\frac{\partial u_{\eta}}{\partial t}-\frac{\beta_{1}(\eta)}{\beta(\eta)} k u_{\eta} \frac{\partial \eta}{\partial t}, \text { then } \\
& \quad \frac{\partial}{\partial x} \int_{z}^{\eta} \frac{\partial w}{\partial t} d z=\frac{\partial u_{\eta}}{\partial t}-\frac{\beta_{1}(\eta)}{\beta(\eta)} k u_{\eta} \frac{\partial \eta}{\partial t}-\frac{\partial u}{\partial t}
\end{aligned}
$$

(4.7).

$$
\begin{gathered}
\frac{\partial u}{\partial t}=-\frac{\partial u_{\eta}}{\partial t}+\frac{\beta_{1}(\eta)}{\beta(\eta)} k u_{\eta} \frac{\partial \eta}{\partial t}+\frac{\partial u}{\partial t} \\
-\frac{1}{2} \frac{\partial}{\partial x}\left(u_{\eta} u_{\eta}+w_{\eta} w_{\eta}\right)-g \frac{\partial \eta}{\partial x}
\end{gathered}
$$

The first term on the right hand side is moved to the left, where as the third term on the right hand side is eliminating each other with the first term on the left hand side.

$$
\begin{aligned}
& \frac{\partial u_{\eta}}{\partial t}=\frac{\beta_{1}(\eta)}{\beta(\eta)} k u_{\eta} \frac{\partial \eta}{\partial t} \\
& -\frac{1}{2} \frac{\partial}{\partial x}\left(u_{\eta} u_{\eta}+w_{\eta} w_{\eta}\right)-g \frac{\partial \eta}{\partial x}
\end{aligned}
$$

Considering that $\frac{\partial}{\partial x} \int_{z}^{\eta} \frac{\partial w}{\partial t} d z$ is formulated using the result of Laplace solution equation coming from the continuity equation, it can be said that there is a role of continuity equation on driving force in equation (4.9) or in other words the momentum equation is controlled by continuity equation.

The momentum equation in equation (4.9) is in the form of surface momentum equation that produces surface velocity of $u_{\eta}$ based on Beji et.al [8]. The equation can be changed to momentum equation for velocity other than surface velocity. In this paper, the equation is changed into equation with calculation velocity equation, i.e. velocity of a depth $z=z_{0}$, where $u\left(z_{0}\right)=U$. Using $z_{0}=-0.4 h$, the velocity of calculation $U$ is similar to the depth-averaged velocity in Airy long wave equation.

$$
\text { Equation (2.3) can be written }
$$

as $u_{\eta}=\frac{\beta(\eta)}{\beta\left(z_{0}\right)} U$, therefore

$\frac{\partial u_{\eta}}{\partial t}=\frac{\beta(\eta)}{\beta\left(z_{0}\right)} \frac{\partial U}{\partial t}+\frac{\beta_{1}(\eta)}{\beta\left(z_{0}\right)} k U \frac{\partial \eta}{\partial t}$ Substituted to equation (4.9),

$\frac{\beta(\eta)}{\beta\left(z_{0}\right)} \frac{\partial U}{\partial t}+\frac{\beta_{1}(\eta)}{\beta\left(z_{0}\right)} k U \frac{\partial \eta}{\partial t}=\frac{\beta_{1}(\eta)}{\beta(\eta)} k u_{\eta} \frac{\partial \eta}{\partial t}$

$-\frac{1}{2} \frac{\partial}{\partial x}\left(u_{\eta} u_{\eta}+w_{\eta} w_{\eta}\right)-g \frac{\partial \eta}{\partial x}$

The equation is divided with $\frac{\beta(\eta)}{\beta\left(z_{0}\right)}, u_{\eta}$ in the first term on the right hand side substitutes $u_{\eta}=\frac{\beta(\eta)}{\beta\left(z_{0}\right)} U$ and the second term is moved to the right hand side, finally:

$$
\frac{\partial U}{\partial t}=-\left(\frac{1}{2} \frac{\partial}{\partial x}\left(u_{\eta} u_{\eta}+w_{\eta} w_{\eta}\right)+g \frac{\partial \eta}{\partial x}\right) \frac{\beta\left(z_{0}\right)}{\beta(\eta)}
$$

This momentum equation will produce horizontal velocity $u$, in a position $z=z_{0}$, i.e. $U$.

\section{V.THE FORMULATION OF WATER SURFACE EQUATION}

Equation for water surface $\frac{\partial \eta}{\partial t}$ is formulated by integrating continuity and energy conservation equation based on Mei [9], where the result of the integration of each equation will produce $\frac{\partial \eta}{\partial t}$. Then, the last equation is the superposition of both $\frac{\partial \eta}{\partial t}$ equations. 
5.1 Equation $\frac{\partial \eta}{\partial t}$ of continuity equation integration

This equation is formulated from continuity equation, i.e. continuity equation integrated to vertical axis $z$, from the bottom of the water $z=-h$ to the surface $z=\eta$.

$\int_{-h}^{\eta} \frac{\partial u}{\partial x} d z+w_{\eta}-w_{-h}=0$ Leibniz rule is applied,

$\int_{z}^{b} \frac{\partial u}{\partial x} d z=\frac{\partial}{\partial x} \int_{a}^{b} u d z-u_{a} \frac{\partial a}{\partial x}+u_{b} \frac{\partial b}{\partial x}$, according

Dean [5], it becomes

$$
\begin{aligned}
& \frac{\partial}{\partial x} \int_{-h}^{\eta} u d z-u_{\eta} \frac{\partial \eta}{\partial x}-u_{-h} \frac{\partial h}{\partial x}+w_{\eta}-w_{-h}=0 \\
& \frac{\partial}{\partial x} \int_{-h}^{\eta} u d z+\frac{\partial \eta}{\partial t}=0 \quad \text { or, } \frac{\partial \eta}{\partial t}=-\frac{\partial}{\partial x} \int_{-h}^{\eta} u d z
\end{aligned}
$$

The kinematic water surface boundary and kinematic water base boundary are applied, where $w_{\eta}=\frac{\partial \eta}{\partial t}+u_{\eta} \frac{\partial \eta}{\partial x}$ and $w_{-h}=-u_{-h} \frac{\partial h}{\partial x}$, and similar terms are added and the result is,

$\frac{\partial}{\partial x} \int_{-h}^{\eta} u d z+\frac{\partial \eta}{\partial t}=0 \quad$ or, $\frac{\partial \eta}{\partial t}=-\frac{\partial}{\partial x} \int_{-h}^{\eta} u d z$

From equation (2.3), $u=\frac{\beta(z)}{\beta\left(z_{0}\right)} U$

$\frac{\partial \eta}{\partial t}=-\frac{\partial}{\partial x} \int_{-h}^{\eta} u d z=-\frac{\partial}{\partial x} \int_{-h}^{\eta} \frac{\beta(z)}{\beta\left(z_{0}\right)} U d z$

$\frac{\partial \eta}{\partial t}=-\frac{\partial \beta_{u H} U}{\partial x}$

where $\beta_{u H}=\frac{\beta_{1}(\eta)-(\alpha-1)}{k \beta\left(z_{0}\right)}$

5.2 Equation $\frac{\partial \eta}{\partial t}$ of the integration of energy conservation equation

[2] is,

Energy conservation equation of Hutahean

$\frac{\partial E_{k}}{\partial t}+\frac{\partial u E_{k}}{\partial x}+\frac{\partial w E_{k}}{\partial z}=0$

where $E_{k}=\frac{u u+w w}{2 g}$. The product of integration of this equation is,

$$
\begin{gathered}
\beta_{E k} \frac{\partial \eta}{\partial t}=-\frac{1}{2 g}\left(\int_{-h}^{\eta} \frac{\beta^{2}(z)}{\beta^{2}\left(z_{0}\right)} d z\right) \frac{\partial U U}{\partial t} \\
-\frac{1}{2 g}\left(\int_{-h}^{\eta} \frac{\beta_{1}^{2}(z)}{\beta_{1}^{2}\left(z_{0}\right)} d z\right) \frac{\partial W W}{\partial t} \\
-\int_{-h}^{\eta} \frac{\partial u E_{k}}{\partial x} d z-\int_{-h}^{\eta} d w E_{k}
\end{gathered}
$$

Where, dimension $\beta_{E k}$ and the right side of the equation are eliminating each other so that $\beta_{E k}$ is non-dimensional number.

$$
\begin{aligned}
\beta_{E k}= & \frac{1}{2 g}\left(\frac{(\beta(2 \eta)+2 \alpha)}{\beta^{2}\left(z_{0}\right)} U U-u_{\eta} u_{\eta}\right) \\
& +\frac{1}{2 g}\left(\frac{(\beta(2 \eta)-2 \alpha)}{\beta_{1}^{2}\left(z_{0}\right)} W W-w_{\eta} w_{\eta}\right)
\end{aligned}
$$

Where $U$ and $W$ are calculation velocity. Equation (5.4) is super imposed with equation (5.2), and is written as equation for $\frac{\partial \eta}{\partial t}$,

$$
\begin{aligned}
&\left(1+\beta_{E k}\right) \frac{\partial \eta}{\partial t}=-\frac{\partial \beta_{u H} U}{\partial x} \\
&-\left(\int_{-h}^{\eta} \frac{\beta^{2}(z)}{\beta^{2}\left(z_{0}\right)} d z\right) \frac{\partial U U}{\partial t}-\left(\int_{-h}^{\eta} \frac{\beta_{1}^{2}(z)}{\beta_{1}^{2}\left(z_{0}\right)} d z\right) \frac{\partial W W}{\partial t} \\
&-\int_{-h}^{\eta} \frac{\partial u E_{k}}{\partial x} d z-\int_{-h}^{\eta} d w E_{k}
\end{aligned}
$$

Equations for integration on the right hand side of the equation (5.6) can be seen in Appendix.

\section{THE RESULT OF THE MODEL}

Momentum and water surface equations are done numerically where spatial differential is done with finite difference method, whereas time differential is done using predictor-corrector method based on numeric integration from Newton-Cote developed by Hutahaean [2]. Then, the numeric model was applied in various cases

\subsection{As Water Surface Equation, just Continuity Equation}

The model is done using sinusoidal wave with a wave period of 8 seconds and amplitude of $0.80 \mathrm{~m}$, at a constant depth of $10 \mathrm{~m}$ and $5 \mathrm{~m}$. At a constant depth of $10 \mathrm{~m}$, the result of the model is presented in Fig. 6.1, where the wave profile is seen deforming toward Cnoidal wave. Part of the wave valley decreases by $0.20 \mathrm{~m}$, where the lowest valley elevation becomes $-0.60 \mathrm{~m}$ and the top of the wave is a little over $0.80 \mathrm{~m}$, 
i.e. $0.86 \mathrm{~m}$. So at this depth of $10 \mathrm{~m}$, there are some wave setups. At a depth of $5 \mathrm{~m}$, the valley elevation is $-0.40 \mathrm{~m}$, whereas the top elevation is $0.94 \mathrm{~m}$. The wave setup that is occurring gets bigger and the Cnoidal profile gets clearer,

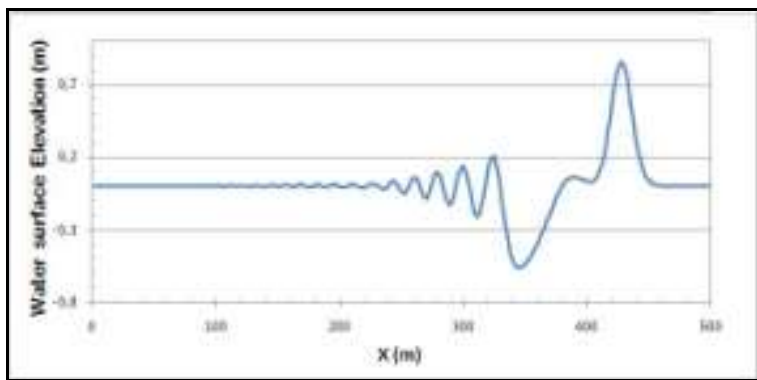

Figure 6.1. The result of the model for a wave with period of 8 seconds, amplitude $0.80 \mathrm{~m}$, constant depth of $10 \mathrm{~m}$

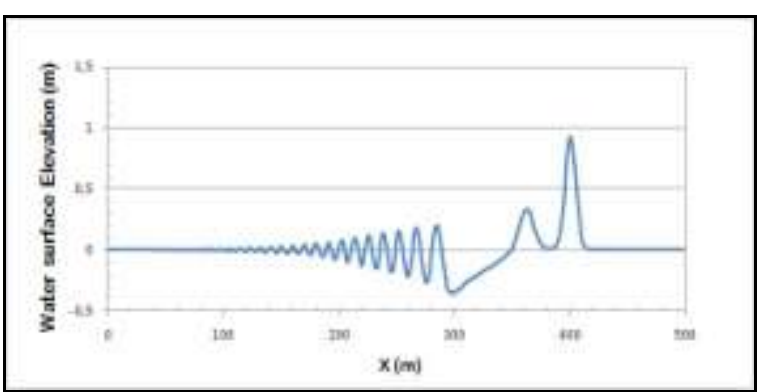

Figure 6.2. The Result of model for a wave with a period of 8 seconds, amplitude $0.80 \mathrm{~m}$, and constant depth of $5 \mathrm{~m}$.

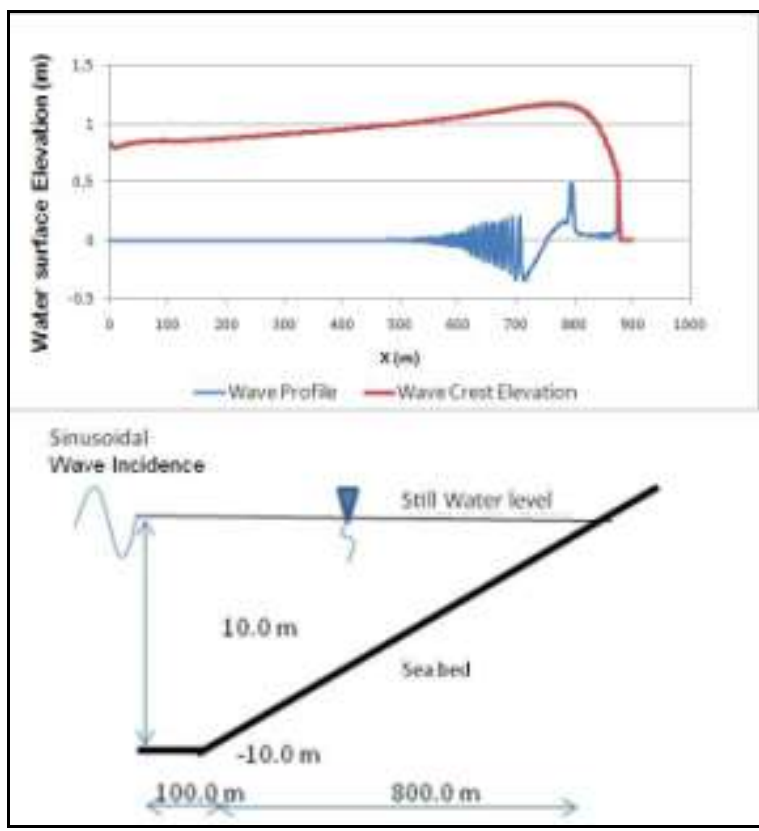

Figure 6.3. The result of model for a wave with a period of 8 seconds, amplitude $0.80 \mathrm{~m}$, changing depth

Then the model was done in the water with not constant depth. Initially, the depth of the water was constant at $10 \mathrm{~m}$, at a distance span of $100 \mathrm{~m}$. Then, the depth changed continuously until a depth of $0.0 \mathrm{~m}$ with a distance span of $800 \mathrm{~m}$. The wave input used is sinusoidal with a period of 8 seconds, amplitude 0.80 $\mathrm{m}$. The results of the model are presented on Fig. 6.2 and Fig. 6.3 which shows the increase of water surface elevation as a result of wave setup, and increase in wave amplitude (shoaling) as a result of the shoaling of the water. Then at the peak elevation of $1.18 \mathrm{~m}$, at a depth of $1.77 \mathrm{~m}$, the water surface elevation declines continuously or breaking taking place. Another phenomenon that can be seen in the very shallow water is that the wave profile becomes fully Cnoidal.

From the work of the model, on constant as well as changing depth, a conclusion can be made that the model contains the phenomena of wave setup, shoaling and breaking, and produces Cnoidal wave profile.

\section{A. As Water Surface Equation, Superimpose Continuity Equation with Energy Conservation Equation}

With similar wave input and bottom of water, as in the previous section, the result of the model is shown on Fig. 6.4 below. The figure shows that there are shoaling and breaking. The breaking started to occur at a depth of $2.68 \mathrm{~m}$, at the peak of elevation or amplitude of $1.07 \mathrm{~m}$, where initially the breaking occurred continuously and then discontinuously.

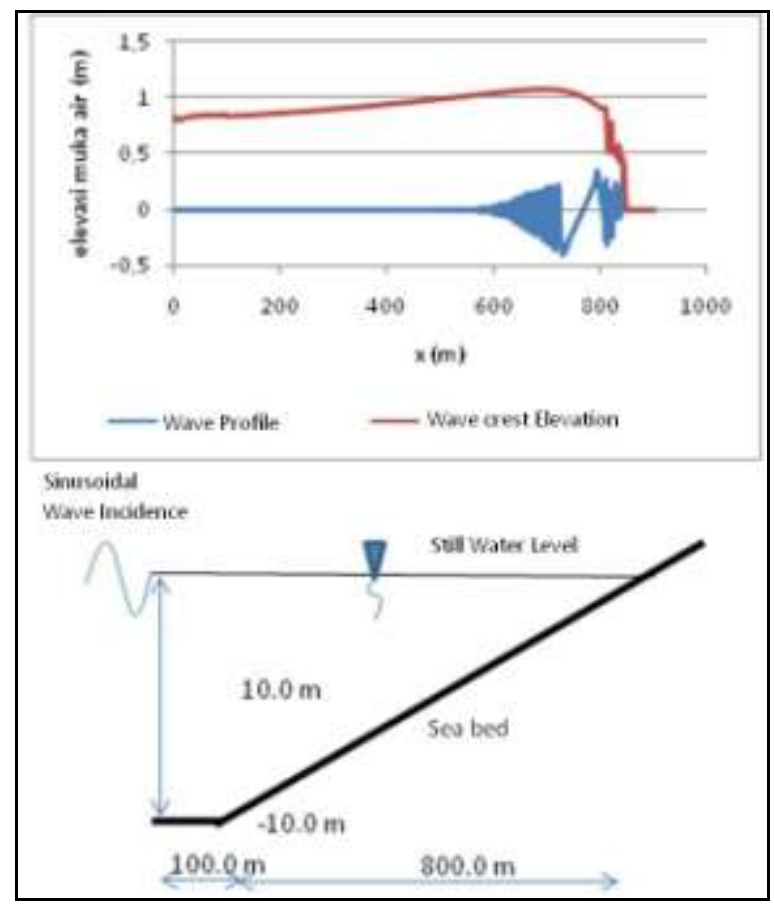

Figure 6.4. The result of the model for a wave with a period of 8 seconds, amplitude of $0.80 \mathrm{~m}$, changing depth, superimpose with Momentum Eq. 
Compare with the previous result, the application of the energy conservation equation on the water surface equation produces model with faster breaking where in the previous result, the breaking started at a depth of $1.77 \mathrm{~m}$, amplitude $1.18 \mathrm{~m}$.

Fig. 6.5 shows the result of the model at a constant depth of $2.70 \mathrm{~m}$, with sinusoidal wave input similar to model at changing depth. The elevation of the top of the wave is $0.96 \mathrm{~m}$ with Cnoidal wave profile. At that depth, at changing depth, the elevation of the top is $1.07 \mathrm{~m}$. So, the component of the increase of wave amplitude as a result of shoaling is more or less $0,11 \mathrm{~m}$. This clarifies that the elevation of top of the wave or the height of wave at shallow water is a result of wave setup and shoaling, with wave setup component that is not small compared with shoaling component.

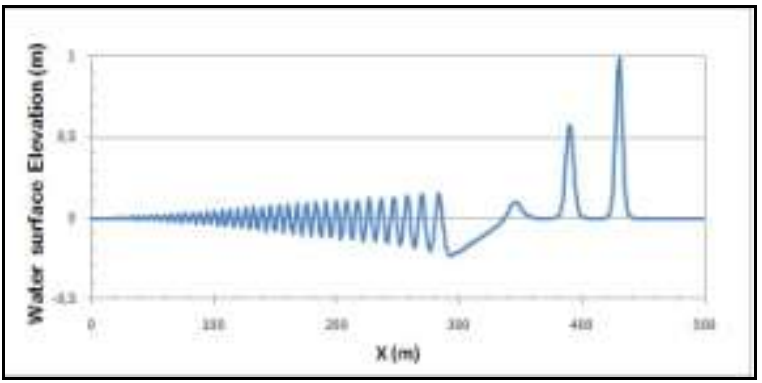

Figure 6.5. The result of the model for a wave with a period of 8 seconds, amplitude of $0.80 \mathrm{~m}$, constant depth of $2.70 \mathrm{~m}$

In addition, the work of energy conservation equation in the water surface equation can model wave unstableness at the shallow water after breaking.

\section{CONCLUSION}

The first conclusion is that the formulation of time series water wave equation using velocity equation as the result of Laplace equation solution simplifies the process of integration of basic equation toward a depth, where equation with calculation velocity variable is produced. The use of calculation velocity concept at time series model caused the model to be done very easily at very shallow water.

The model developed in this paper can stimulate wave setup, shoaling and breaking well and produce Cnoidal wave profile. In other words, the model can stimulate various natural phenomena occurring in a wave on its journey to shallow water.

By working on the velocity equation as the result of Laplace equation solution at the formulation of momentum equation and water surface equation, there is wave number $k$ at the momentum equation and water surface equation. In order for the number to correspond with the equation that is used, it is better to use wave number that is calculated from the equation of kinematic water surface boundary and complete momentum equation, with methods developed by Hutahaean [3], and [4] and Lin et.al. [10].

By using velocity equation as the result of Laplace equation solution at the formulation of governing equation, the velocity produced by momentum equation will be more controlled by continuity equation, considering Laplace equation originated from continuity equation.

The work on energy conservation equation on water surface equation causes the wave to be faster in breaking than without energy conservation equation. In addition, wave instability after breaking is also visible on the water surface equation which is a superposition between continuity equation and energy conservation equation.

\section{APPENDIX: INTEGRATION OF ENERGY CONSERVATION EQUATION}

Energy conservation equation, i.e. equation (5.3), is multiplied with $d z$ and integrated with depth,

$$
\int_{-h}^{\eta} \frac{\partial E_{k}}{\partial t} d z+\int_{-h}^{\eta} \frac{\partial u E_{k}}{\partial x} d z+\int_{-h}^{\eta} \partial w E_{k}=0
$$

On the integration of this energy conservation equation, the concept of calculation velocity $U$ and $W$ is done, where $U=u\left(x, z_{0}, t\right)$ and $W=u\left(x, z_{0}, t\right)$ where the relation of this velocity with velocity at any position of $z$ is, $\frac{u}{U}=\frac{\beta(z)}{\beta\left(z_{0}\right)}$ and $\frac{w}{W}=\frac{\beta_{1}(z)}{\beta_{1}\left(z_{0}\right)}$.

a. $\int_{-h}^{\eta} \frac{\partial E_{k}}{\partial t} d z=\frac{1}{2 g} \int_{-h}^{\eta} \frac{\partial}{\partial t}\left(u^{2}+w^{2}\right) d z$

a.1. $\int_{-h}^{\eta} \frac{\partial u^{2}}{\partial t} d z$

Based on the concept of calculation velocity, where $u u=\frac{\beta(z) \beta(z)}{\beta^{2}\left(z_{0}\right)} U U$, then

$\int_{-h}^{\eta} u u d z=U U \int_{-h}^{\eta} \frac{\beta(z) \beta(z)}{\beta^{2}\left(z_{0}\right)} d z$. The work on Leibniz rule,

$\int_{-h}^{\eta} \frac{\partial u u}{\partial t} d z=\frac{\partial}{\partial t} \int_{-h}^{\eta} u u d z-u_{\eta} u_{\eta} \frac{\partial \eta}{\partial t}$. The work on depth-averaged velocity 


$$
\begin{aligned}
& \int_{-h}^{\eta} \frac{\partial u u}{\partial t} d z=\frac{\partial}{\partial t}\left(U U \int_{-h}^{\eta} \frac{\beta^{2}(z)}{\beta^{2}\left(z_{0}\right)} d z\right)-u_{\eta} u_{\eta} \frac{\partial \eta}{\partial t} \\
& \int_{-h}^{\eta} \frac{\partial u u}{\partial t} d z=\left(\int_{-h}^{\eta} \frac{\beta^{2}(z)}{\beta^{2}\left(z_{0}\right)} d z\right) \frac{\partial U U}{\partial t} \\
& +U U \frac{\partial}{\partial t} \int_{-h}^{\eta} \frac{\beta^{2}(z)}{\beta^{2}\left(z_{0}\right)} d z-u_{\eta} u_{\eta} \frac{\partial \eta}{\partial t} \\
& \int_{-h}^{\eta} \frac{\partial u u}{\partial t} d z=\left(\int_{-h}^{\eta} \frac{\beta^{2}(z)}{\beta^{2}\left(z_{0}\right)} d z\right) \frac{\partial U U}{\partial t} \\
& +\left(\frac{(\beta(2 \eta)+2 \alpha)}{\beta^{2}\left(z_{0}\right)} U U-u_{\eta} u_{\eta}\right) \frac{\partial \eta}{\partial t}
\end{aligned}
$$

where,

$$
\begin{aligned}
& \int_{-h}^{\eta} \beta^{2}(z) d z=\left(\frac{1}{2 k}\left(\beta_{1}(2 \eta)-\left(\alpha^{2}-1\right)\right)+2 \alpha H\right) \\
& \text { and } \frac{\partial}{\partial t} \int_{-h}^{\eta} \beta^{2}(z) d z=(\beta(2 \eta)+2 \alpha) \frac{\partial \eta}{\partial t} \\
& \text { a.2. } \int_{-h}^{\eta} \frac{\partial w^{2}}{\partial t} d z
\end{aligned}
$$

From the concept of calculation velocity,

$$
\text { then, } w w=\frac{\beta_{1}(z) \beta_{1}(z)}{\beta_{1}^{2}\left(z_{0}\right)} W W \text { then }
$$$$
\int_{-h}^{\eta} w w d z=W W \int_{-h}^{\eta} \frac{\beta(z) \beta(z)}{\beta^{2}\left(z_{0}\right)} d z
$$

The work on Leibniz integration

$$
\int_{-h}^{\eta} \frac{\partial w w}{\partial t} d z=\frac{\partial}{\partial t} \int_{-h}^{\eta} w w d z-w_{\eta} w_{\eta} \frac{\partial \eta}{\partial t}
$$

Substitute relation of calculation velocity,

$$
\begin{gathered}
\int_{-h}^{\eta} \frac{\partial w w}{\partial t} d z=\frac{\partial}{\partial t}\left(W W \int_{-h}^{\eta} \frac{\beta(z) \beta(z)}{\beta^{2}\left(z_{0}\right)} d z\right)-w_{\eta} w \\
\int_{-h}^{\eta} \frac{\partial w w}{\partial t} d z=\left(\int_{-h}^{\eta} \frac{\beta_{1}^{2}(z)}{\beta_{1}^{2}\left(z_{0}\right)} d z\right) \frac{\partial W W}{\partial t} \\
+W W \frac{\partial}{\partial t} \int_{-h}^{\eta} \frac{\beta_{1}^{2}(z)}{\beta_{1}^{2}\left(z_{0}\right)} d z-w_{\eta} w_{\eta} \frac{\partial \eta}{\partial t} \\
\int_{-h}^{\eta} \frac{\partial w w}{\partial t} d z=\left(\int_{-h}^{\eta} \frac{\beta_{1}^{2}(z)}{\beta_{1}^{2}\left(z_{0}\right)} d z\right) \frac{\partial W W}{\partial t}
\end{gathered}
$$

$$
+\left(\frac{(\beta(2 \eta)-2 \alpha)}{\beta_{1}^{2}\left(z_{0}\right)} W W-w_{\eta} w_{\eta}\right) \frac{\partial \eta}{\partial t}
$$

Where

$\int_{-h}^{\eta} \beta_{1}^{2}(z) d z=\left(\frac{1}{2 k}\left(\beta_{1}(2 \eta)-\left(\alpha^{2}-1\right)\right)-2 \alpha H\right)$, then

$\frac{\partial}{\partial t} \int_{-h}^{\eta} \beta_{1}^{2}(z) d z=(\beta(2 \eta)-2 \alpha) \frac{\partial \eta}{\partial t}$

b. $\int_{-h}^{\eta} \frac{\partial u E_{k}}{\partial x} d z=\frac{1}{2 g} \int_{-h}^{\eta} \frac{\partial}{\partial x}\left(u\left(u^{2}+v^{2}+w^{2}\right)\right) d z$

b.1. $\int_{-h}^{\eta} \frac{\partial u u^{2}}{\partial x} d z$

$$
\begin{aligned}
\frac{u u^{2}}{U U^{2}} & =\frac{\beta^{3}(z)}{\beta^{3}\left(z_{0}\right)} \text { or } u u^{2}=\frac{\beta^{3}(z)}{\beta^{3}\left(z_{0}\right)} U U^{2} \text { then } \\
\int_{-h}^{\eta} u u^{2} d z & =U U^{2} \int_{-h}^{\eta} \frac{\beta^{3}(z)}{\beta^{3}\left(z_{0}\right)} d z
\end{aligned}
$$

The work on Leibniz rule

$\int_{-h}^{\eta} \frac{\partial u u^{2}}{\partial x} d z=\frac{\partial}{\partial x} \int_{-h}^{\eta} u u^{2} d z-u_{\eta} u_{\eta}^{2} \frac{\partial \eta}{\partial x}-u_{-h} u_{-h}^{2} \frac{\partial h}{\partial x}$

The work on the concept of calculation

velocity

$$
\begin{aligned}
\int_{-h}^{\eta} \frac{\partial u u^{2}}{\partial x} d z & =\frac{\partial}{\partial x}\left(U U^{2} \int_{-h}^{\eta} \frac{\beta^{3}(z)}{\beta^{3}\left(z_{0}\right)} d z\right)-u_{\eta} u_{\eta}^{2} \frac{\partial \eta}{\partial x} \\
& -u_{-h} u_{-h}^{2} \frac{\partial h}{\partial x}
\end{aligned}
$$

Where

$$
\int_{-h}^{\eta} \beta^{3}(z) d z=\frac{1}{3 k}\left(\beta_{1}(3 \eta)-\left(\alpha^{3}-1\right)\right)
$$




$$
\begin{aligned}
\int_{-h}^{\eta} \frac{\partial u w^{2}}{\partial x} d z & =\frac{\partial}{\partial x} \int_{-h}^{\eta} u w^{2} d z-u_{\eta} w_{\eta}^{2} \frac{\partial \eta}{\partial x} \\
& -u_{-h} w_{-h}^{2} \frac{\partial h}{\partial x}
\end{aligned}
$$

Substitute calculation velocity,

$$
\begin{aligned}
\int_{-h}^{\eta} \frac{\partial u w^{2}}{\partial x} d z & =\frac{\partial}{\partial x}\left(U W^{2} \int_{-h}^{\eta} \frac{\beta(z) \beta_{1}^{2}(z)}{\beta\left(z_{0}\right) \beta_{1}^{2}\left(z_{0}\right)} d z\right) \\
& -u_{\eta} w_{\eta}^{2} \frac{\partial \eta}{\partial x}-u_{-h} w_{-h}^{2} \frac{\partial h}{\partial x}
\end{aligned}
$$

Where, $\beta(z) \beta_{1}^{2}(z)=\beta(3 z)+\alpha \beta_{1}(z)$

$$
\begin{aligned}
& \int_{-h}^{\eta} \beta(z) \beta_{1}^{2}(z) d z=\frac{1}{3 k}\left(\beta_{1}(3 \eta)-\left(\alpha^{3}-1\right)\right) \\
& +\frac{\alpha}{k}(\beta(\eta)-(\alpha+1)) \\
& \text { d. } \int_{-h}^{\eta} d w E_{k}=\frac{1}{2 g}\left(w_{\eta}\left(u_{\eta}^{2}+v_{\eta}^{2}+w_{\eta}^{2}\right)\right. \\
& \left.\quad-w_{-h}\left(u_{-h}^{2}+v_{-h}^{2}+w_{-h}^{2}\right)\right)
\end{aligned}
$$

In general, the integration of energy conservation equation can be written as,

$$
\begin{aligned}
& \beta_{E k} \frac{\partial \eta}{\partial t}=-\frac{1}{2 g}\left(\int_{-h}^{\eta} \frac{\beta^{2}(z)}{\beta^{2}\left(z_{0}\right)} d z\right) \frac{\partial U U}{\partial t} \\
& -\frac{1}{2 g}\left(\int_{-h}^{\eta} \frac{\beta_{1}^{2}(z)}{\beta_{1}^{2}\left(z_{0}\right)} d z\right) \frac{\partial W W}{\partial t} \\
& -\int_{-h}^{\eta} \frac{\partial u E_{k}}{\partial x} d z-\int_{-h}^{\eta} d w E_{k}
\end{aligned}
$$

where

$$
\begin{gathered}
\beta_{E k}=\frac{1}{2 g}\left(\frac{(\beta(2 \eta)+2 \alpha)}{\beta^{2}\left(z_{0}\right)} U U-u_{\eta} u_{\eta}\right) \\
+\frac{1}{2 g}\left(\frac{(\beta(2 \eta)-2 \alpha)}{\beta_{1}^{2}\left(z_{0}\right)} W W-w_{\eta} w_{\eta}\right)
\end{gathered}
$$

In this case, $\beta_{E k}$ looks as if it had meter dimension, but the dimension is eliminating each other with the right side of the equation.

\section{Acknowledgements}

The authors acknowledge that this paper publication is supported by Program of Research and Public Service and Innovation (P3MI) of Coastal Engineering Research Groups, Faculty of Civil and
Environmental Engineering, Institut Teknologi Bandung (ITB) for fiscal year 2017.

\section{REFERENCES}

[1] Fenton J.D., Non Linear wave theories, The Sea-Ocean Engineering Science, Part A, B. Le Mehaute \& D.M. Hanes (eds), Vol 9, Wiey, New York, 1990

[2] Hutahaean, S., Wave Dynamics Modeling with Derivation of Conservation Energy Equation -in Indonesian, Jurnal Teknik Sipil, Fakultas Teknik Sipil dan Lingkungan, ITB Indonesia, Volume 14 No.1, January, 2007.

[3] Hutahaean, S., Wave Refraction Model Using non Linier Equation -in Indonesian, Jurnal Infrastruktur dan Lingkungan Binaan, Fakultas Teknik Sipil dan Lingkungan, ITB Indonesia, Volume III, No. 2, 2007.

[4] Hutahaean, S., Refraction-difraction of Water Wave Model Due to Bathymetry -In Indonesian, Jurnal Teknik Sipil, Fakultas Teknik Sipil dan Lingkungan, ITB Indonesia, Volume 15, No.2, August 2008.

[5] Dean, Robert G., and Dalrymple, Water Wave Mechanics for Engineers and Scientists. Prentice-Hall, Englewood Cliffs, New Jersey, 1984.

[6] Hutahaean, S., Deformation of Sinusoidal Wave Become Cnoidal Wave -in Indonesian, Jurnal Teknik Sipil, Fakultas Teknik Sipil dan Lingkungan, ITB, Indonesia, Volume 18 No.2, August 2011.

[7] Hutahaean,S, Wave Model Using Hydrodynamics Pressure based on Continuity Equation for Accelerated Fluid.-in Indonesian, Jurnal Teknik Sipil, Fakultas Teknik Sipil dan Lingkungan, ITB Indonesia, Volume 19 No.2, August 2012.

[8] Beji S. and Nadaoka K., A Formal Derivation and Numerical Modeling of Improved Boussinesq Equation for Varying Depth, Ocean Engineering, Vol. 23 (8), pp 691-704, 1996.

[9] Mei C.C., The Applied Dynamics of Ocean Surface Waves, Advanced Series on Ocean Engineering I, Chapter 11, World Scientific, 1989.

[10] C. Y. Lin, M. Wu, J. A. Bloom, I. J. Cox, and M. Miller, Rotation, scale, and translation resilient public watermarking for images, IEEE Trans. Image Process., vol. 10 , no. 5, pp. 767-782, May 2001 